\title{
Estudo químico de Zeyheria montana M. (bolsa-de-pastor)
}

\author{
Jácome, R.L.R. P.1*; Raslan, D. S.2; Wagner, H.3; Oliveira, A.B. de ${ }^{1}$ \\ 'Laboratório de Farmacognosia, Faculdade de Farmácia; 2Departamento de Química, Instituto \\ de Ciências Exatas, Universidade Federal de Minas Gerais; Instituto de Biologia \\ Farmacêutica, Universidade de Munique, Alemanha.
}

\begin{abstract}
RESUMO: Do extrato hexânico das raízes de Zeyheria montana M., uma Bignoniácea conhecida como bolsa-de-pastor, empregada popularmente contra doenças de pele, foram isolados os seguintes compostos: lapachol, $\alpha$-lapachona, desidro- $\alpha$-lapachona, 4-hidróxi$\alpha$-lapachona, $\beta$-sitosterol, ácido esteárico e uma mistura de ácidos graxos. Estas substâncias foram identificadas por métodos espectrométricos usuais e/ou comparação com amostras autênticas. No cromatograma do extrato etanólico, obtido por CLAE, foram identificados os picos correspondentes às naftoquinonas isoladas e ao ácido esteárico. A ocorrência de naftoquinonas nas raízes de $Z$. montana e a atividade antimicrobiana comprovada para 0 lapachol e a $\alpha$-lapachona justificam o uso popular desta espécie.
\end{abstract}

Unitermos: Zeyheria montana, Bignoniaceae, naftoquinonas.

ABSTRACT: Zeyheria montana M. (Bignoniaceae) is a widespread species in the Cerrado area of Minas Gerais, Brazil, and is popularly known as bolsa-de-pastor. In the Brazilian tradicional medicine its roots are used for the treatment of skin diseases. Several species of Bignoniaceae contain naphthoquinones, and lapachol was previously reported in the stem wood of $Z$. digitalis. This paper describes the isolation of three naphtoquinones from $Z$. montana stem barks, namely $\alpha$-lapachone, dehydro- $\alpha$-lapachone and 4-hydroxy- $\alpha$-lapachone, besides lapachol, stearic acid and B-sitosterol. Furthermore, we report the HPLC fingerprint for the species, which allowed the identification of the isolated compounds, except $B$-sitosterol. Since the in vitro antimicrobial activity of lapachol and $\alpha$-lapachone has been previously described, the presence of these naphthoquinones in the roots of $Z$. montana corroborates its popular use for the treatment of skin diseases.

Key words: Zeyheria montana, Bignoniaceae, naphtoquinones.

INTRODUÇÃO: Zeyheria montana M. é uma Bignoniácea nativa do Brasil, conhecida como bolsa-de-pastor, cujas raízes são usadas, popularmente, contra doenças da pele (Pio Corrêa, 1931). Sua madeira foi estudada anteriormente com a sinonímia de $Z$. digitalis (Vell.) Hoehne (Gentry, 1992), e de seus extratos benzênico e etanólico foram isolados zeyherol, D-glicose, ácido vanílico, ácido verátrico e uma única naftoquinona, o lapachol (Silveira et al., 1975). As naftoquinonas são freqüentes nesta família, a exemplo de $Z$. tuberculosa (Weinberg et al., 1976), Tabebuia serratifolia (Raslan, 1981), T. incana (Oliveira et al., 1993), T. ochracea (Zani et al., 1991), T. serratifolia (Miraglia, 1991) e Lundia densiflora(Ferreira, 1986).

Revista Brasileira de Farmacognosia 11 (1) (2001) 5-12

ISSN: 0102-695X 
O lapachol (I), constituinte químico freqüente e abundante nesta família, teve diversas atividades biológicas e farmacológicas comprovadas experimentalmente, tais como atividades antibacteriana (Houghton et al., 1997) e antiinflamatória (Almeida et al., 1990). Também apresentou, em humanos, atividade antineoplásica (Santana et al., 1980), confirmando os resultados dos experimentos realizados em animais portadores de neoplasias malignas.<smiles>CC(C)=CCC1=C(O)C(=O)c2ccccc2C1=O</smiles>

Uma outra Bignoniácea freqüentemente confundida com $Z$. montana é a $Z$. tuberculosa (Vell.) Bur., de cuja madeira foram anteriormente isoladas quatro naftoquinonas: lapachol (I), $\alpha$-lapachona (II), desidro- $\alpha$-lapachona (III) e 4-hidróxi- $\alpha$-lapachona (IV) (Weinberg et al., 1976). A presença de naftoquinonas em $Z$. montana e $Z$. tuberculosa poderia justificar o uso medicinal dessas espécies.<smiles>CC1(C)CCC2=C(O1)C(=O)c1ccccc1C2=O</smiles><smiles>CC1(C)C=CC2=C(O1)C(=O)c1ccccc1C2=O</smiles><smiles>CC1(C)C=C(O)C2=C(O1)C(=O)c1ccccc1C2=O</smiles>

Em trabalho anterior, relatou-se a quantificação de lapachol, $\alpha$-lapachona e desidro- $\alpha$ lapachona no extrato etanólico das raízes de Z. montana, além do perfil cromatográfico, obtido por cromatografia líquida de alta eficiência (CLAE) de fase reversa. No cromatograma desse extrato foram identificados, ainda, os picos correspondentes a 4-hidróxi- $\alpha$-lapachona e ao ácido esteárico (Jácome et al., 1999).

\section{MATERIAIS E MÉTODOS}

\section{Material Botânico}

A planta foi coletada durante a primavera de 1993, em São Gotardo, Minas Gerais, Brasil, e foi identificada por J. L. Pedersoli. Uma exsicata encontra-se depositada no Herbário do Museu de História Natural, Belo Horizonte, MG, sob o $n^{\circ} 4466$.

\section{Extração e isolamențo das substâncias}

Ao extrato etanólico (12 g) das raízes de $Z$. montana (122 g), obtido por percolação, adicionou-se etanol-água (7:3) e foram realizadas partições sucessivas com $n$-hexano e clorofórmio, obtendo-se as frações hexânica, clorofórmica, hidroetanólica e um precipitado que se formou por concentração parcial da fração hexânica. O precipitado $(0,58 \mathrm{~g})$ e a parte solúvel em hexano $(2,1 \mathrm{~g})$ foram submetidos à cromatografia em coluna de gel de sílica (CCS) eluindo-se com hexano $(3 \mathrm{~L})$, hexano/diclorometano $(5 \mathrm{~L})$, diclorometano $(4 \mathrm{~L})$, diclorometano/metanol $(13 \mathrm{~L})$ e metanol $(1 \mathrm{~L})$. Estes procedimentos estão esquematizados na Figura 1. 
A fração oleosa 1-5, obtida a partir da CCS do material solúvel em hexano, foi submetida à saponificação, por refluxo com solução aquosa de $\mathrm{KOH}$ a $6 \%$. Em seguida, após extração com éter etílico, a fração aquosa foi acidificada com $\mathrm{HCl}$ e extraída com éter etílico. A fração etérea foi esterificada com metanol/ $\mathrm{H}_{2} \mathrm{SO}_{4}$. A mistura dos ésteres metílicos obtida foi analisada por cromatografia gasosa usando-se como padrões: tridecanoato de metila, miristato de metila, pentadecanoato de metila, palmitato de metila, margarato de metila, linoleato de metila, 11,12oleato-linolenato de metila, estearato e monodecanoato de metila.

As frações 8-20 (hexano), 131-145 (hexano/diclorometano), 184-216 e 340-349 (hexano/ diclorometano) foram submetidas à cromatografia em camada delgada de gel de sílica G (CCD) preparativa, usando-se tolueno: acetato de etila (93:7) como eluente (Wagner et al., 1984).

Dessa forma, foram isolados o lapachol (140 mg) a partir de $F$ 8-20 (0,220 g), a desidro- $\alpha$ -lapachona (7 mg) de F 131-145 (0,052 g), a $\alpha$-lapachona (40 mg) de F 184-216 (0,250 g) e a 4-hidróxi- $\alpha$-lapachona ( $7 \mathrm{mg}$ ) de $F 340-349(0,038 \mathrm{~g})$, cuja purificação final foi realizada por CLAE de fase reversa preparativa com mistura de acetonitrila e água (80:20).

A fração oleosa 23-28 (40 mg/hexano) quando adicionada de acetonitrila forneceu um precipitado branco que foi identificado como ácido esteárico.

A fração $349-363(0,053 \mathrm{~g})$ precipitou em metanol fornecendo um sólido branco que foi separado por filtraçāo e identificado como ß-sitosterol $(4 \mathrm{mg})$.

A fração 481-488, eluída com metanol, forneceu um precipitado branco, solúvel em metanol/ água (2:1) que, quando cromatografado em placa de gel de sílica $\mathrm{G}$ (eluente $\mathrm{CHCl}_{3}: \mathrm{MeOH}: \mathrm{H}_{2} \mathrm{O}$ / 64:40:8; revelador difenilamina-anilina-ácido fosfórico $85 \%$ ), possibilitou a identificação de sacarose, glicose e rafinose, por comparação com padrōes de açúcares.

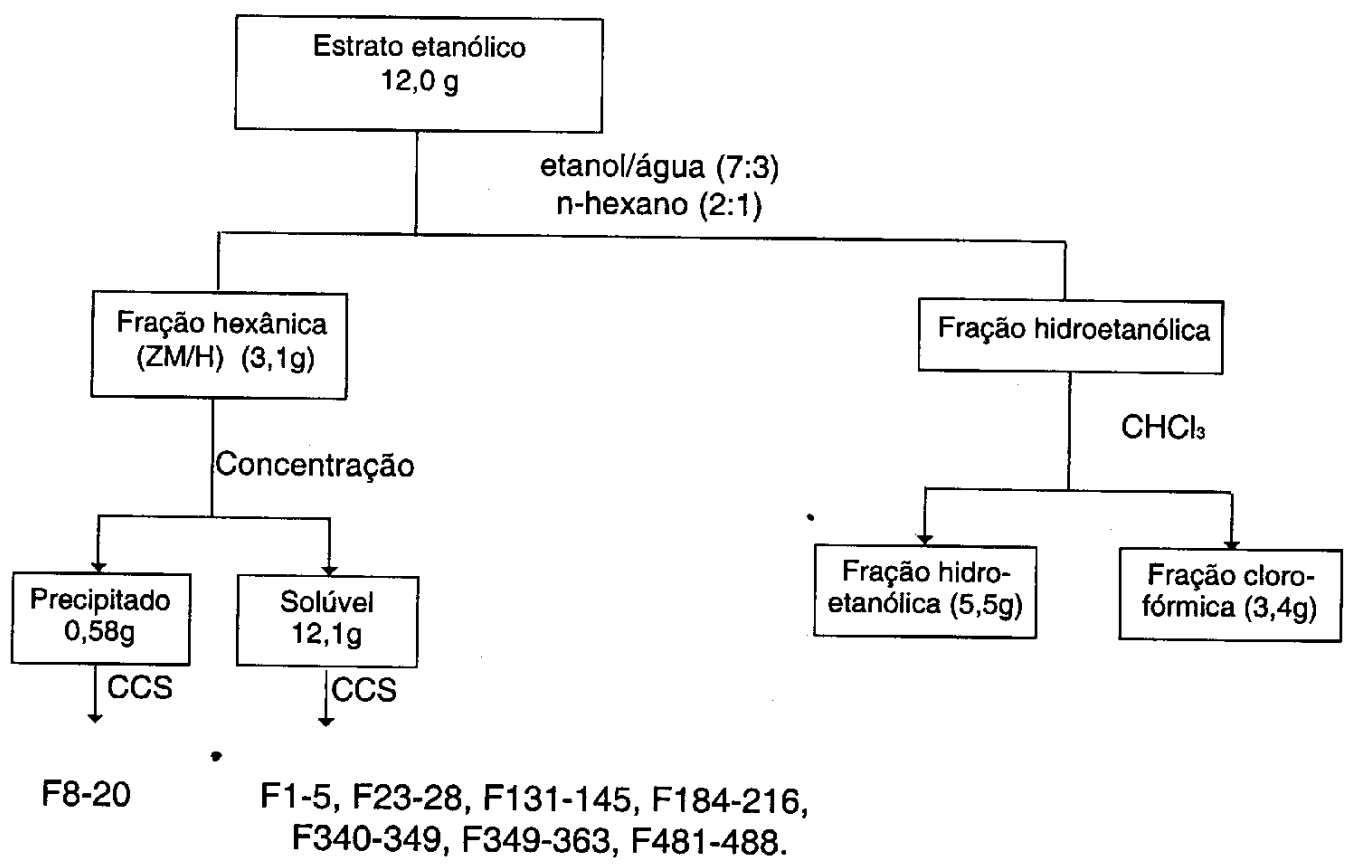

FIGURA 1. Partiçāo do extrato etanólico das raízes de Zeyheria montana entre solventes imiscíveis e cromatografia em coluna de gel de sílica (CCS) do material solúvel em hexano e do precipitado. 
Dados físico-químicos e espectrométricos das substâncias isoladas

Lapachol (F 8-20, ZM/H) (I)

Sólido cristalino, amarelo, p.f. $139^{\circ} \mathrm{C}\left(\mathrm{CH}_{2} \mathrm{Cl}_{2}\right)$ (Thompson, 1971). IV (v máx., $\left.\mathrm{KBr}, \mathrm{cm}^{-1}\right)$ : 3350 , $1680,1650,1600$ e 1500. UV ( $\lambda$ máx., nm): Obtido em CLAE (acetonitrila/água) 251, 278, 333. $\mathrm{RMN}^{1} \mathrm{H}\left(\mathrm{CDCl}_{3}, 360 \mathrm{MHz}, \delta\right): 1,7(3 \mathrm{H}, \mathrm{s}), 1,8(3 \mathrm{H}, \mathrm{s}), 3,3(2 \mathrm{H}, \mathrm{d}, J=7,4 \mathrm{~Hz}), 5,2(1 \mathrm{H}, \mathrm{t}, J=7,4 \mathrm{~Hz})$, $8,05(1 \mathrm{H}, \mathrm{d}, J=8,8 \mathrm{~Hz}), 8,15(1 \mathrm{H}, \mathrm{d}, J=8,8 \mathrm{~Hz}), 7,7(1 \mathrm{H}, \mathrm{t}, J=8,8 \mathrm{~Hz}), 7,8(1 \mathrm{H}, \mathrm{t}, J=8,8 \mathrm{~Hz}), 7,3$ $(1 \mathrm{H}, \mathrm{sl}) . \mathrm{RMN}{ }^{13} \mathrm{C}\left(\mathrm{CDCl}_{3}, 100 \mathrm{MHz}, \delta\right)$ : Tabela 1 (McDonald et al., 1977; Ferreira et al., 1987).

Q-lapachona (F 184-216, ZM/H) (II)

Sólido cristalino amarelo: p. f. $114{ }^{\circ} \mathrm{C}\left(\mathrm{CH}_{2} \mathrm{Cl}_{2}\right)$ (Thompson, 1971). IV (v max., $\left.\mathrm{KBr}, \mathrm{cm}^{-1}\right): 1680$, $1640,1600,1580$; UV ( $\lambda$ máx., $\mathrm{nm}$ ): Obtido em CLAE (acetonitrila/água) 250, 280, 330. RMN ${ }^{1} \mathrm{H}$ $\left(\mathrm{CDCl}_{3}, 360 \mathrm{MHz}, \delta\right): 1,45(6 \mathrm{H}, \mathrm{s}) ; 1,85(2 \mathrm{H}, \mathrm{t}, J=6,6 \mathrm{~Hz}) ; 2,65(2 \mathrm{H}, \mathrm{t}, J=6,6 \mathrm{~Hz}) ; 7,7(2 \mathrm{H}, \mathrm{m}) ; 8,1$ $(2 \mathrm{H}, \mathrm{m})$. EM (IE) m/z (int.rel. \%): $242\left(\mathrm{M}^{+}\right), 227(100), 199(16), 159(52) ; 102(30) ; 76(30)$. RMN ${ }^{13} \mathrm{C}\left(\mathrm{CDCl}_{3}, 100 \mathrm{MHz}, \delta\right): 78,1 ; 26,5 ; 26,5 ; 31,4 ; 16,7 ; 126,3 ; 133,8 ; 132,9 ; 126,3 ; 132,0 ; 131,0 ;$ 180,$0 ; 184,4 ; 154,6 ; 120,1$ (Tabela 1) (McDonald et al., 1977; Ferreira et al., 1987).

Desidro- $\alpha$-lapachona (F 131-145, ZM/H) (III)

Sólido cristalino, alaranjado, p.f. $140^{\circ} \mathrm{C}\left(\mathrm{CH}_{2} \mathrm{Cl}_{2}\right)$ (Thompson, 1971). IV (v máx., $\left.\mathrm{KBr}, \mathrm{cm}^{-1}\right)$ : 1685 , 1650, 1600, 1570. UV ( $\lambda$ máx., nm): Obtido em CLAE (acetonitrila/água) 340, 280, 260. EM (IE) $\mathrm{m} / \mathrm{z}$ (int.rel. \%): $240\left(\mathrm{M}^{+}, 20 \%\right) ; 225$ (100), $212(12), 197(40), 104(20), 76(20)$. RMN ${ }^{~} \mathrm{H}\left(\mathrm{CDCl}_{3^{\prime}}\right.$ $360 \mathrm{MHz}, \delta): 1,6(6 \mathrm{H}, \mathrm{s}), 5,7(\mathrm{H}, \mathrm{d}, J=10 \mathrm{~Hz}), 6,6(\mathrm{H}, \mathrm{d}, J=10 \mathrm{~Hz}), 7,7(2 \mathrm{H}, \mathrm{m}), 8,09(\mathrm{H}, \mathrm{d}, J=7,8$ $\mathrm{Hz}), 8,11(\mathrm{H}, \mathrm{d}, J=7,8 \mathrm{~Hz})$. RMN ${ }^{13} \mathrm{C}\left(\mathrm{CDCl}_{3}, 100 \mathrm{MHz}\right.$, d): Tabela 1 (McDonald et al., 1977; Ferreira et al., 1987).

\section{4-hidróxi- $\alpha$-lapachona (F 340-349, ZM/H) (IV)}

Sólido esverdeado, amorfo, p.f. $118-120^{\circ} \mathrm{C}\left(\mathrm{CH}_{2} \mathrm{Cl}_{2}\right)$. IV (u máx., $\left.\mathrm{KBr}, \mathrm{cm}^{-1}\right): 3500,1685,1645$, 1570, 1600. EM (IE) $\mathrm{m} / \mathrm{z}$ (int.rel. \%): $258\left(\mathrm{M}^{+}, 15 \%\right), 203(51), 176$ (45), $146(52), 105$ (43), 83 (100), $56(20)$. RMN ${ }^{1} \mathrm{H}\left(\mathrm{CDCl}_{3}, 360 \mathrm{MHz}, \delta\right): 1,4(3 \mathrm{H}, \mathrm{s}), 1,6(3 \mathrm{H}, \mathrm{s}), 2,25(1 \mathrm{H}, \mathrm{d}, J=6,4 \mathrm{~Hz}), 2,75$ $(1 \mathrm{H}, \mathrm{d}, J=6,3 \mathrm{~Hz}), 3,9(\mathrm{H}, \mathrm{sl}), 5,0(\mathrm{H}, \mathrm{t}, J=6,3 \mathrm{~Hz}), 7,7(2 \mathrm{H}, \mathrm{m}), 8,08(1 \mathrm{H}, \mathrm{dd}, J=7,2 \mathrm{e} 1,2 \mathrm{~Hz})$, $8,12(1 \mathrm{H}, \mathrm{dd}, J=7,2$ e $1,2 \mathrm{~Hz})$. RMN ${ }^{13} \mathrm{C}\left(\mathrm{CDCl}_{3}, 100 \mathrm{MHz}, \delta\right)$ : Tabela 1 (McDonald et al., 1977; Ferreira et al., 1987).

\section{B-Sitosterol (F 349-363, ZM/H)}

Sólido branco, p.f. $140^{\circ} \mathrm{C}$. ( Dey, 1991). IV (u máx., $\left.\mathrm{KBr}, \mathrm{Cm}^{-1}\right): 3500$, 3300, 2980, 2880, 1640. EM (IE) $\mathrm{m} / \mathrm{z}$ (int.rel. \%): $414\left(\mathrm{M}^{+}, 100 \%\right), 399$ (83), 396 (55), 381 (33), 329 (40), 303 (58), 273 (49), 255 (100), 231 (39), 213 (74).

\section{Preparação dos ésferes metílicos de ZM/H - F 1-5}

Óleo incolor. Condições de CG: coluna SE 54, metilfenil-silicona 5\% (30 m x 0,32mm) FID: miristato de metila, palmitato de metila, margarato de metila, estearato de metila, oleato de metila, linoleato de metila e linolenato de metila. Padrões: tridecanoato de metila, miristato de metila, pentadecanoato de metila, palmitato de metila, margarato de metila, linoleato de metila, 11,12-oleato-linolenato de metila, estearato de metila, monodecanoato de metila. 
Ácido esteárico (F 23-28, ZM/H)

Sólido branco, p.f. $76^{\circ} \mathrm{C}$ (acetonitrila) (Markley, 1947). IV (v máx., $\mathrm{KBr}, \mathrm{cm}^{-1}$ ): 2920, 2840, 1710, 1465, 710. EM (IE) m/z (int.rel. \%): $284\left(\mathrm{M}^{+}, 30\right), 256(100), 241(10), 227(15), 213(40), 199(10)$, $185(50), 171(28), 157(25), 143(15), 129(19), 115$ (40), 97 (5), 73 (30), 60 (43), $55(68), 43(97)$, $41(100)$.

\section{RESULTADOS E DISCUSSÃO}

Os espectros no infravermelho com absorções em torno de 1600, 1650 e $1680 \mathrm{~cm}^{-1}$, associados à cor amarela e às colorações alaranjada e amarelo-esverdeada sugeriram a natureza naftoquinônica de quatro das substâncias isoladas. Os pontos de fusão e comparação com padrões através de CCD e CLAE sugeriram tratar-se do lapachol (I), da $\alpha$-lapachona (II), da desidro- $\alpha$ lapachona (III) e da 4-hidróxi- $\alpha$-lapachona (IV). A confirmação da identificação destas substâncias resultou da análise dos espectros no UV, RMN'H, $\mathrm{RMN}^{13} \mathrm{C}$ e de massas.

O espectro de RMN'H do lapachol mostrou dois singletos em $\delta 1,7$ e 1,8 e a integração de cada um deles para $3 \mathrm{H}$, indicou tratar-se dos 2 grupos metila. Os outros sinais, um dubleto em $\delta 3,3(\mathrm{~J}=7,4 \mathrm{~Hz})$, correspondente a dois hidrogênios, e um tripleto centrado em $\delta 5,2(\mathrm{~J}=7,4 \mathrm{~Hz})$ correspondente a um hidrogênio, evidenciaram a presença do grupo metileno $\left(-\mathrm{CH}_{2}-\mathrm{CH}=\mathrm{C}\right)$. Dois dubletos centrados em $\delta 8,05 e \delta 8,15(\mathrm{~J}=7,6 \mathrm{~Hz})$ foram atribuídos aos hidrogênios peri às carbonilas $(\mathrm{H}-5$ e H-8) e dois tripletos centrados em $\delta 7,7$ e $\delta 7,8(\mathrm{~J}=7,5 \mathrm{~Hz})$ aos dois hidrogênios $H-6$ e H-7. Em $\delta 7,3$ observou-se um singleto correspondente ao hidrogênio do grupo hidroxila.

$O$ espectro de RMN'H da $\alpha$-lapachona apresentou um singleto em $\delta 1,45$, correspondente aos 6 hidrogênios dos 2 grupos metila. Dois tripletos centrados em $\delta 1,85$ e $\delta 2,65(\mathrm{~J}=6,6 \mathrm{~Hz})$, com integração correspondente a dois hidrogênios cada um, confirmaram a presença de dois grupos metilenos vicinais $\left(\mathrm{CH}_{2}-\mathrm{CH}_{2}\right)$. O tripleto em $\delta 2,65$ pode ser atribuído aos hidrogênios do carbono 4, que são mais desprotegidos pela carbonila do anel vizinho. Observou-se, ainda, dois multipletos centrados em $\delta 7,7$ e $\delta 8,1$ referentes, cada um, a 2 hidrogênios do anel aromático.

No espectro de $\mathrm{RMN}^{1} \mathrm{H}$ da desidro- $\alpha$-lapachona, observou-se um singleto em $\delta 1,6$ correspondente aos 6 hidrogênios dos 2 grupos metila. Dois dubletos centrados em $\delta 5,7$ e $\delta 6,6$ $(\mathrm{J}=10,0 \mathrm{~Hz})$ evidenciaram os hidrogênios olefínicos do anel pirano, sendo que, ao $\mathrm{H}-4 \mathrm{deve}$ corresponder o maior deslocamento químico, devido à sua desproteção pela carbonila do anel quinônico. Dois hidrogênios aromáticos, $\mathrm{H}-6$ e H-7, originaram um multipleto centrado em 87,7 e os dubletos centrados em $\delta 8,09$ e $8,11(\mathrm{~J}=6,0 \mathrm{~Hz})$ correspondem a $\mathrm{H}-5$ e H-8.

Quanto ao espectro de RMN'H da 4-hidróxi- $\alpha$-lapachona, os singletos em $\delta 1,4$ e $\delta 1,6$ referem-se aos dois grupos metila. Dois dubletos duplos centrados em $\delta 2,05(\mathrm{~J}=6,4 \mathrm{~Hz})$ e 2,15 $(\mathrm{J}=6,3 \mathrm{~Hz}$ ), correspondentes a 1 hidrogênio cada, foram atribuídos ao grupo metileno do carbono 3 do anel pirano. O hidrogênio do carbono 4 originou o tripleto centrado em $\delta 5,0(\mathrm{~J}=6,3 \mathrm{~Hz})$. Dos hidrogênios aromáticos, os dois em peri às carbonilas $(\mathrm{H}-5$ e H-8) originaram dubletos duplos, parcialmente superpostos, centrados em $\delta 8,08$ e $\delta 8,12(\mathrm{~J}=5,2$ e $1,2 \mathrm{~Hz})$. Para $\mathrm{H}-6$ e $\mathrm{H}-7$ observouse um multipleto centrado em $\delta 7,7$. O singleto largo em d3,9 evidenciou o hidrogênio hidroxílico.

As atribuições dos sinais nos espectros de $\mathrm{RMN}^{13} \mathrm{C}$ (Tabela 1) estão de acordo com aquelas descritas na literatura (McDonald et al., 1977; Ferreira et al., 1987). 
TABELA 1. Dados de RMN ${ }^{13} \mathrm{C}$ das naftoquinonas isoladas das raízes de Zeyheria montana.

\begin{tabular}{c|c|c|c|c}
\hline Carbonos & F8-20 (I) & F 184-216 (II) & $131-145(I I I)$ & $340-349(\mathrm{IV})$ \\
\hline 1 & 184,5 & 184,4 & $\cdot 181,9$ & 186,0 \\
\hline 2 & 123,5 & 120,1 & 117,8 & 120,3 \\
\hline 3 & 152,7 & 154,6 & 152,4 & 154,0 \\
\hline 4 & 181,7 & 180,0 & 179,9 & 180,0 \\
\hline 5 & 126,7 & 126,3 & 126,2 & 126,6 \\
\hline 6 & 132,7 & 132,9 & 133,2 & 133,4 \\
\hline 7 & 135,0 & 133,8 & 134,0 & 134,3 \\
\hline 8 & 126,2 & 125,9 & 126,2 & 126,0 \\
\hline 9 & 133,8 & 132,0 & 131,5 & 131,8 \\
\hline 10 & 129,5 & 131,1 & 131,5 & 131,1 \\
\hline 11 & 25,7 & 16,7 & 115,4 & 60,0 \\
\hline 12 & 119,6 & 31,4 & 130,9 & 39,5 \\
\hline 13 & 133,0 & 78,1 & 80,4 & 79,7 \\
\hline 14 & 22,6 & 26,5 & $28,3^{*}$ & $26,7^{*}$ \\
\hline 15 & 17,8 & 26,5 & $29,7^{*}$ & $27,0^{*}$ \\
\hline
\end{tabular}

*Sinais intercabiáveis

A fração 1-5, de aspecto oleoso, é uma mistura de ácidos graxos, conforme reação com bromocresol, em CCD. Esta fração foi submetida à reação de saponificação/esterificação e os tempos de retenção dos constituintes foram comparados com aqueles dos padrões de ésteres metílicos, por cromatografia gasosa.

O precipitado, obtido da fração 349-363, foi identificado como B-sitosterol pelos espectros no IV e de massas, além de comparação direta com amostra autêntica.

Os açúcares sacarose, glicose e rafinose foram identificados por comparação, através de CCD, com padrões autênticos.

Dentre as naftoquinonas isoladas, o lapachol corresponde ao pico de maior área, com tempo de retenção igual a $28,8 \mathrm{~min}$, mas a substância com maior área de pico no extrato, nas condições de análise, foi identificada como ácido esteárico, com tempo de retenção igual a 45,57 min (Figura 2).

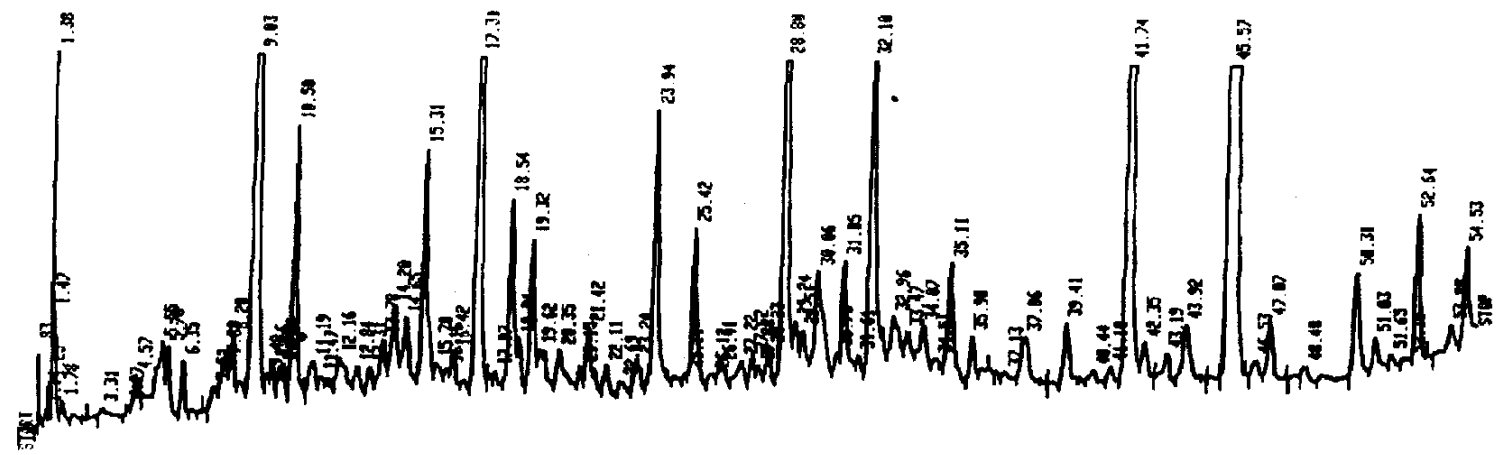

FIGURA 2. Cromatograma, obtido por CLAE, do extrato bruto das raízes de Zeyheria montana. Identificação dos picos : $1=4$-hidróxi- $\alpha$-lapachona $(17,31 \mathrm{~min}), 2=\alpha$-lapachona $(23,94 \mathrm{~min}), 3=$ desidro- $\alpha$-lapachona $(25,42 \mathrm{~min}), \quad 4=$ lapachol $(28,8 \mathrm{~min})$ e 5 = ácido esteárico (45,57 min). Condições cromatográficas: acetonitrila/água (10:90) passando para (90:10) em $55 \mathrm{~min}$. 


\section{CONCLUSÃo}

Foram isoladas e identificadas seis substâncias a partir do extrato hexânico, obtido das raízes de $Z$. montana: lapachol, desidro- $\alpha$-lapachona, $\alpha$-lapachona, 4-hidróxi- $\alpha$-lapachona, ß-sitosterol e ácido esteárico, além de uma fração graxa convertida em ésteres metílicos (miristato, palmitato, margarato, estearato, oleato, linoleato e linolenato de metila) caracterizada por CG/EM. Uma mistura de açúcares foi identificada como sendo sacarose, glicose e rafinose.

O perfil cromatográfico do extrato etanólico das raízes de $Z$. montana foi obtido através de CLAE de fase reversa. O cromatograma do extrato etanólico mostra picos correspondentes às naftoquinonas, ao ácido esteárico e a várias outras substâncias que poderão ser isoladas das frações clorofórmica e hidroetanólica, em trabalhos posteriores.

\section{REFERÊNCIAS BIBLIOGRÁFICAS}

ALMEIDA, E.R.; SILVA FILHO, A.A.; SANTOS, E.R.; LOPES, C.A.C. Antiinflammatory action of lapachol. J. Ethnopharmacol., 29: 239-241, 1990.

DEY, P.M.; HARBORNE, J.B. Methods in plant biochemistry terpenoids. London: Academic Press. 1991. v.7.

FERREIRA, C.A.C.; FERREIRA, V.F.; PINTO, A.V.; LOPES, R.S.C.; PINTO, M. C.R.; SILVA, A.J.R. ${ }^{13} \mathrm{C}$ NMR Spectra of natural products Part 5 - Naphthopyrandiones and naphthofurandiones. An. Acad. Bras. Cienc., 59 (1/2): 5-8, 1987.

FERREIRA, D.T. Estudo químico de Lundia densiflora Pyr. DC e síntese de diidropiranonaftoquinonas. Belo Horizonte: UFMG / ICEX, 1986. 196p. Tese de Doutorado.

GENTRY, A.H. Flora neotropica. New York: The New York Botanical Garden, 1992. 370p.

HOUGHTON, P.J.; MAT ALI, R.; AZIZOL, M. Antimicrobial activity of extracts of some Bignoniaceae from Malaysia. Pharm. Pharmacol. Lett., 7 (2/3): 96-98, 1997.

JÁCOME, R.L.R.P.; RASLAN, D.S.; MULLER, A.; WAGNER, H.; OLIVEIRA, A.B. Análise de naftoquinonas em extratos brutos de Zeyheria montana (bolsa-de-pastor). Quím. Nova, 22 (2):175-177, 1999.

MARKLEY, K.S. Fatty acids. New York: Interscience, 1947.

MCDONALD, I.A.; SIMPSON, T.J.; SIERAKOWSKI, A.F. ${ }^{13} \mathrm{C}$ NMR Spectral studies of some naturally occurring quinones and related compounds. Aust. J. Chem., 30: 1727-1234, 1977.

MIRAGLIA, M.C.M. Estudo químico de Tabebuia serratifolia (Vahl.) Nichols (Bignoniaceae) e síntese de piranonaftoquinonas, furanonaftoquinonas e antraquinonas. Belo Horizonte: UFMG / ICEX, 1991. 326p. Tese de Doutorado.

OLIVEIRA, A.B.; RASLAN, D.S.; OLIVEIRA, G.G.; MAIA, J.G.S. Lignans and naphthoquinones

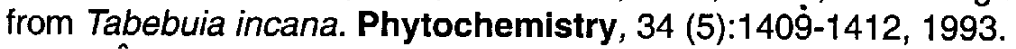

PIO CORREA, M. Dicionário das plantas úteis do Brasil e das exóticas cultivadas, Rio de Janeiro: Ministério da Agricultura/IBDF, 1931. v. 2, p.215.

RASLAN, D.S. Constituintes químicos de Tabebuia serratifolia(Vahl.) Nichols. Belo Horizonte: UFMG / ICEX, 1981, 114p. Dissertação de Mestrado.

SANTANA, C.F.; LINS, L.J.P.; ASFORA, J.J.; MELO, A.M.; LIMA, O.G.; D'ALBUQUERQUE I. L. Primeiras observações com o emprego do lapachol em pacientes humanos portadores de neoplasias malignas. Rev. Inst. Antib., 20 (1/2): 61-68, 1980/1.

SILVEIRA, J.C.; GOTTLIEB, O.R.; DE OLIVEIRA, G.G. Zeyherol, a dilignol from Zeyheria digitalis. Phytochemistry, 14: 1829-1830, 1975.

THOMPSON, R.H. Naturally occurring quinones. 2. ed. London: Academic Press, 1971.

WAGNER, H.; BLADT, S.; ZGAINSKI, E.M. Plant drug analysis. New York: Springer-Verlag, 1984.

WEINBERG, M.L.D.; GOTTLIEB, O.R.; OLIVEIRA, G.G. Naphthoquinones from Zeyheria tuberculosa. Phytochemistry, 15: 570-570, 1976. 
ZANI, C. L.; OLIVEIRA, A.B.; OLIVEIRA, G.G. Furanonaphthoquinones from Tabebuia ochracea. Phytochemistry, 30 (7): 2379-2381, 1991.

Autor para correspondência:

Profa. Dra. Rose Lisieux R. Paiva Jácome Laboratório de Farmacognosia

Faculdade de Farmácia

Universidade Federal de Minas Gerais

Avenida Olegário Maciel, 2360

30180-112 - Belo Horizonte - MG

E-mail: lisięux $\oplus$ dedalus.lcc.ufmg.br 